\title{
Successful High Dose Glucocorticoid Treatment for Subacute Neuromyelitis Optica with Systemic Lupus Erythematosus
}

\author{
Noriko HagIWARA****, Kazunori TOYODA*, Takeshi UWATOKO*, \\ Kotaro YASUMORI**, Setsuro IBAYASHI*** and Yasushi OKADA*
}

\begin{abstract}
A 54-year-old Japanese woman with a 6-year history of systemic lupus erythematosus (SLE) was admitted to our hospital suffering from acute blindness in her right eye. Her condition recovered after steroid pulse therapy, however, 18 months later she suffered from nuchal pain for 2 weeks after which right hemiparesis with urinary incontinence developed. A spinal magnetic resonance imaging (MRI) revealed cord swelling from $\mathrm{C} 2$ to $\mathrm{C} 7$. She was diagnosed with neuromyelitis optica (NMO) and intravenous steroid administrations were immediately commenced. Her condition promptly improved. This case was unique because the steroid treatment was quite effective for this case of myelitis, which had passed the acute phase. We supposed that, because most of the lesion was not necrotic or demyelinated, but rather showed edematous change caused by vasculitis based on autoimmune pathogenesis, the symptoms progressed rather gradually and improved promptly in response to glucocorticoid treatment.
\end{abstract}

(Internal Medicine 44: 998-1001, 2005)

Key words: systemic lupus erythematosus, neuromyelitis optica, glucocorticoid, vasculitis, magnetic resonance imaging

\section{Introduction}

Neuromyelitis optica (NMO) is characterized by optic neuritis and acute myelitis with unknown etiology, and is one of the most serious manifestations of systemic lupus erythematosus (SLE). Although a combination of aggressive treatments consisting of high doses of corticosteroid, immunosuppressive drugs and plasma exchange have been tried in acute stage patients, the prognosis is often unfavorable. Herein, we report a case of NMO complicated with SLE. An association with vasculitis caused by humoral mechanisms was strongly suggested as the cause of the prompt glucocorticoid treatment response despite a delay of 4 weeks from the onset of symptoms until the start of treatment.

\section{Case Report}

A 54-year-old Japanese woman diagnosed with SLE and treated for 6 years with oral prednisolone was admitted to our hospital complaining of sudden visual loss in her right eye. SLE diagnosis was based on the following symptoms satisfying the revised criteria recommended by the American College of Rheumatology $(1,2)$ : malar rash, photosensitivity, non-erosive symmetrical arthritis, and a positive antinuclear antibody (ANA) test. As an accompanying symptom, she had Raynaud's phenomenon. On admission, her right visual acuity was reduced to light perception only. She was diagnosed by an ophthalmologist as having retrobulbar optic neuritis and started on a course of intravenous methylprednisolone injections at $1,000 \mathrm{mg}$ daily for three days followed by oral prednisolone. Her visual acuity gradually improved to normal.

Eighteen months later, she started to feel nuchal pain, although she had no history of preceding infection, cervical trauma or intervention therapy including radiation. Two weeks later, she suffered from progressing right hemiparesis

\footnotetext{
From the Departments of *Cerebrovascular Disease and **Neuroradiology, Cerebrovascular Center and Clinical Research Institute, National Kyushu Medical Center, Fukuoka and ***the Department of Medicine and Clinical Science, Graduate School of Medical Sciences, Kyushu University, Fukuoka

Received for publication December 17, 2004; Accepted for publication March 9, 2005

Reprint requests should be addressed to Dr. Noriko Hagiwara, the Department of Medicine and Clinical Science, Graduate School of Medical Sciences, Kyushu University, 3-1-1 Maidashi, Higashi-ku, Fukuoka 812-8582
} 
and urinary incontinence. Although she received traction treatment for her neck in a local hospital, her condition gradually deteriorated. During this period, she did not take medicine except for prednisolone. She was introduced to our hospital 29 days after the onset of nuchal pain.

On admission, her blood pressure was 140/90 $\mathrm{mmHg}$, her pulse rate was regular at 80 beats per minute, and her body temperature was $36.5^{\circ} \mathrm{C}$. Her fingers were slightly sclerotic without scar and had purpura. She did not have proximal or other sclerotic changes. On neurological examination, she was alert and well oriented. Her pupils were round and isocoric, and she showed a sluggish light reflex in her right eye. Her right limbs were flaccid and almost completely paralytic with increased deep tendon reflexes and a positive Babinski's reflex, and her left limbs were slightly paretic. Superficial sensations were reduced down the right side of her body.

On immunological testing, she tested positive for ANA (1 : 160, homogeneous, speckled type), and negative for anti-ds DNA, SS-A, SS-B, Sm, U1-RNP, Scl-70, centromere and cardiolipin antibodies. Her serum complements and immune complex (anti-C1q antibody method) were within the normal range as was her erythrocyte sedimentation rate. Her Creactive protein was negative and her blood cell counts, serum chemistry, and urinary tests were all normal.

A brain magnetic resonance imaging (MRI) taken on the hospitalization day was normal, but a spinal MRI taken on the same day revealed cord swelling and a high intensity lesion from $\mathrm{C} 2$ to $\mathrm{C} 7$ on the T2-weighted images (Fig. 1A). On an axial image, the high intense signal expanded to the anterior and right lateral columns (Fig. 1B). There was no enhancement after administration of gadolinium contrast. These lesions did not coincide with the vascular territory. Spinal angiography did not reveal a tumor or vascular abnormality.

Based on these results and the previous history of optic neuritis, we made a diagnosis of NMO and started administration of intravenous betamethasone at $8 \mathrm{mg}$ per day. In addition, $800 \mathrm{ml}$ glycerol per day was also administered to reduce the spinal cord swelling. Six hours after the first administration of betamethasone, the patient could move her fingers slightly, and by day 2 , she could slightly raise her right limbs and felt a need to urinate. On day 7 , she could raise her right limbs and hold them there for several seconds, and her urinary incontinence was almost completely cured. A T2-weighted sagittal MRI taken on day 7 showed reduced cord swelling with a remaining high intense signal from $\mathrm{C} 2$ to C5 (Fig. 1C). On an axial image, the anterior column lesion had almost completely disappeared (Fig. 1D). Cerebrospinal fluid (CSF) examined on day 8 had a normal appearance with the pressure of $75 \mathrm{~mm}$ water. It contained 1.3 white blood cells (WBC) $/ \mathrm{mm}^{3}$, and normal protein and glucose (40 and $78 \mathrm{mg} / \mathrm{dl}$, respectively). IL-6, IgG index and myelin basic protein in the CSF were also within the normal range $(2.9 \mathrm{pg} / \mathrm{ml}$ (normal; <4.0), and 0.37 (normal; <0.70), and $3.5 \mathrm{mg} / \mathrm{ml}$ (normal; <4.0), respectively). An oligoclonal
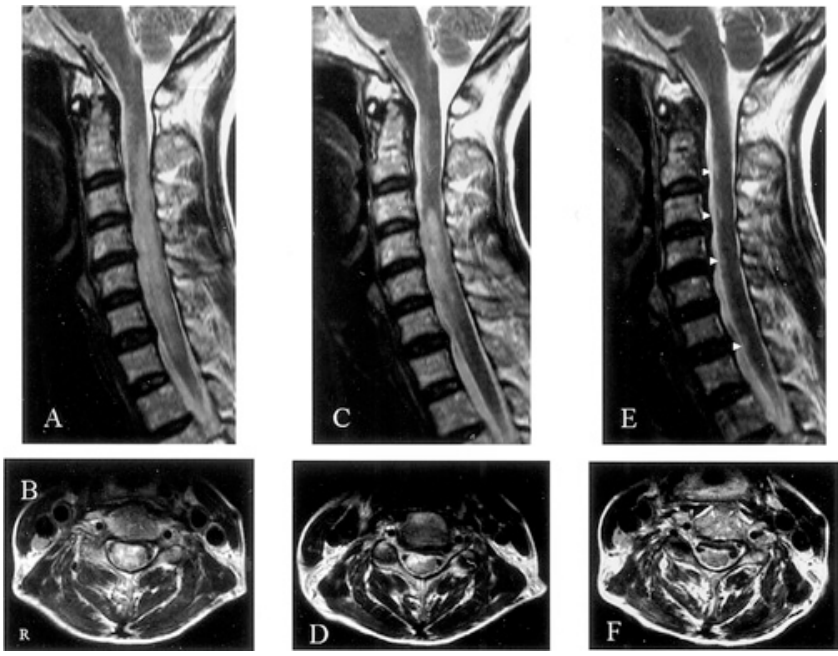

Figure 1. T2-weighted magnetic resonance image of the cervical spine. (A) The high intense lesion with severe spinal swelling extended from $\mathrm{C} 2$ to $\mathrm{C} 7$ seen on admission. (B) Axial image on the $\mathrm{C} 5$ level showed diffuse high intensity in the bilateral anterior and posterior columns and right lateral column. The high intensity area diminished $\mathbf{1 0}$ days after treatment $(\mathrm{C}, \mathrm{D})$, and appeared faint 3 months later (E, F).

band was not detected. Cytology showed no atypical cells and CSF and blood cultures showed no organisms.

Intravenous betamethasone was tapered off according to the improved clinical symptoms and MRI findings, and replaced by $60 \mathrm{mg}$ of oral prednisolone daily. The prednisolone dosage was tapered every 3 days. The glycerol dosage was also tapered down. One month after admission, the patient could walk by herself and a MRI revealed that the cord swelling had disappeared and only a spotty high intensity area in the ventral area remained (Fig. 1E, F). She was discharged without any neurological deficits and has been free from any recurrent signs for more than 2 years (Fig. 2).

\section{Discussion}

NMO is characterized by optic neuritis and acute myelitis of unknown etiology $(3,4)$, and is one of the most serious and rare manifestations of SLE. Previously, most patients who developed NMO during the course of SLE had an unfavorable prognosis and showed a poor response to steroids or immunosuppressive drugs despite rapid diagnosis and prompt aggressive treatment (5-9). In particular, myelitis in SLE often occurs at the cervical level and intensive immunosuppressive therapy causes fatal complications such as severe infection or pulmonary embolism (10). Previous studies have stressed the limited therapeutic time window for severe myelitis in SLE; one report recommended early aggressive treatment within 5 days (10) while another recommended that complete recovery required treatment within 24 hours of symptom onset (11). Contrarily, our patient showed a prompt 


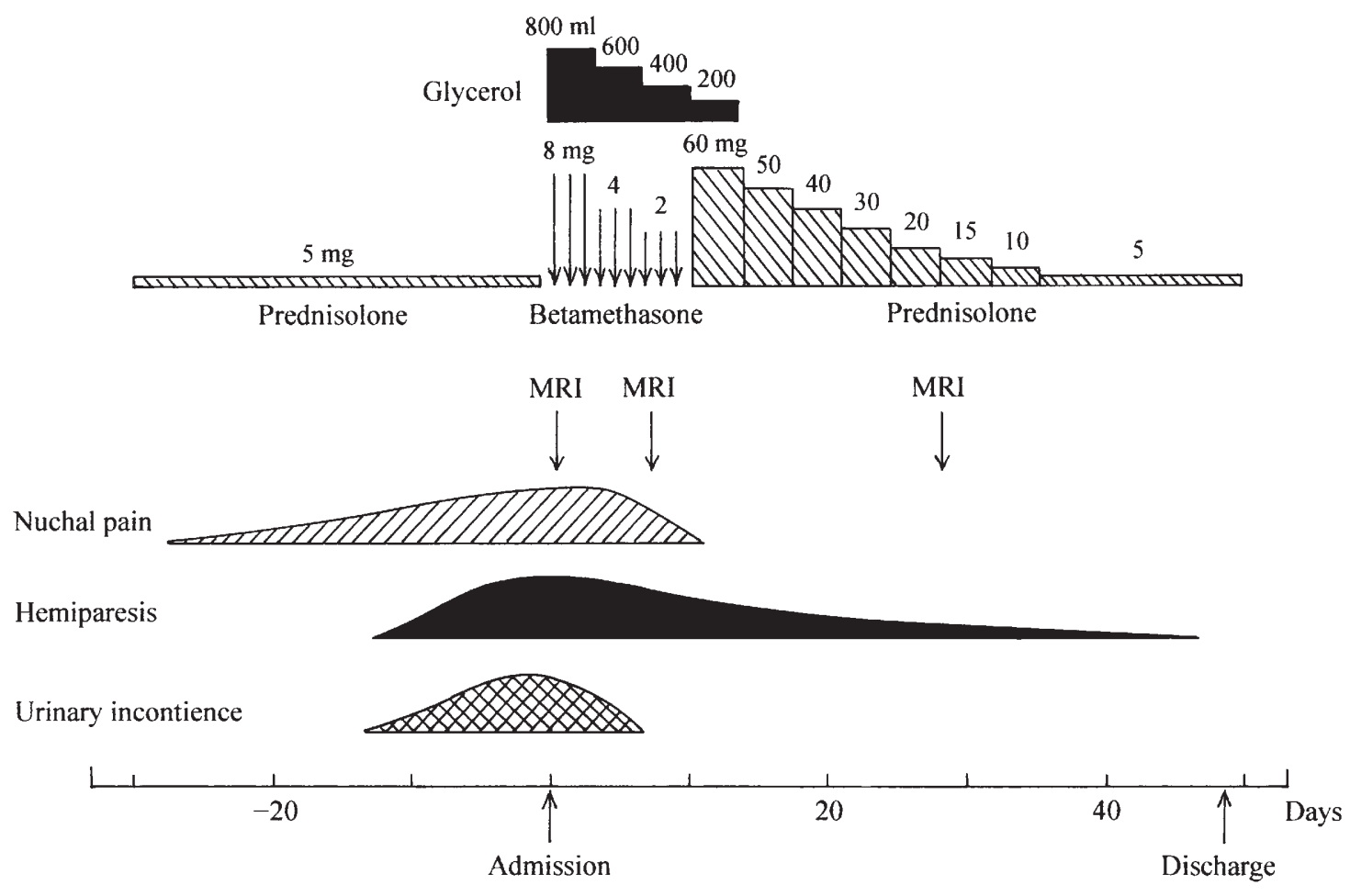

Figure 2. Clinical course.

reaction to intravenous steroid administration despite the 29day interval from the onset of symptoms to treatment.

In addition to the classical pathological findings of the spinal cord in NMO, including extensive demyelination, fulminate necrosis, cavitation and hyalinization of mediumsized arteries (12), Lucchinetti et al (13) found a uniform colocalization of immunoglobulin, C9 neoantigen (a marker of complement-mediated tissue injury) and activated macrophages at the sites of vessel damage, suggesting that the CNS vasculature might be an early and specific target of the disease. Andrianakos et al (11) also clarified prominent vasculitis with ischemic change based on 12 autopsy cases of transverse myelopathy in SLE patients. Futhermore, the circulating autoantibodies and immune complex frequently observed in NMO patients support the hypothesis that humoral mechanisms work as an indicator for the development and relapse of this disease $(12,13)$. We speculated that a vasculitic edema of the spinal cord, instead of necrosis or an ischemic infarct, was the main pathology of the present patient, judging from the lack of inflammatory change, the lack of contrast enhancement on the MRI, and the prompt reactivity to steroid treatment. If there was a chance for spontaneous recovery, it may have strongly been accelerated by the use of corticosteroids. Thus, aggressive steroid treatment should be attempted for SLE patients with NMO, even if the acute stage has passed or inflammatory changes cannot be detected.

Recently, Wingerchuk et al (14) classified the clinical course of NMO into two types namely monophasic or relapsing, and clarified the features of each type through observations of 71 NMO patients. According to their classification, the present patient met the features of relapsing NMO, such as female dominance, older age at onset, longer intervals between optic neuritis and myelitis, unilateral optic neuritis, and the presence of systemic autoimmune disorders. In SLE patients, because the disease activity sometimes does not parallel CNS involvement (15) such as optic neuritis and myelitis, there is a risk of misdiagnosis with such complications.

To prevent relapse, a symptomatical, radiological, and serological follow-up is necessary for our patient. Nuchal and back pain occasionally precede other neurological symptoms of myelitis, and might be a warning sign of relapse. MRI was available for NMO diagnosis in our patient, and in previous reports they seem to have been useful for follow-up observations (16). Especially in the present case, the high intense signal on T2-weighted images tended to parallel the effect of glucocorticoid treatment. Even for patients in remission, we should radiologically examine atrophy or cavitation of the spinal cord and optic nerve as further late complications (13, 17).

\section{References}

1) Tan EM, Cohen AS, Fries JF, et al. The 1982 revised criteria for the classification of systemic lupus erythematosus. Arthritis Rheum 25: 


\section{Glucocorticoid Treatment for NMO with SLE}

1271-1277, 1982.

2) Hochberg MC. Updating the American College of Rheumatology revised criteria for the classification of systemic lupus erythematosus. Arthritis Rheum 40: 1725, 1997.

3) de Seze J, Stojkovic T, Ferriby D, et al. Devic's neuromyelitis optica: clinical, laboratory, MRI and outcome profile. J Neurol Sci 197: 5761, 2002.

4) O'Riordan JI, Gallagher HL, Thompson AJ, et al. Clinical, CSF, and MRI findings in Devic's neuromyelitis optica. J Neurol Neurosurg Psychiatry 60: 382-387, 1996.

5) Gibbs AN, Moroney J, Foley-Nolan D, O'Connell PG. Neuromyelitis optica (Devic's syndrome) in systemic lupus erythematosus: a case report. Rheumatology 41: 470-471, 2002.

6) Bonnet F, Mercie P, Morlat P, et al. Devic's neuromyelitis optica during pregnancy in a patient with systemic lupus erythematosus. Lupus 8: 244-247, 1999.

7) Margaux J, Hayem G, Meyer O, Kahr MF. Systemic lupus erythematosus with optical neuromyelitis (Devic's syndrome). A case with a 35year follow-up. Rev Rheum Engl Ed 66: 102-105, 1999.

8) Kinney EL, Berdoff RL, Rao NS, Fox LM. Devic's syndrome and systemic lupus erythematosus: a case report with necropsy. Arch Neurol 36: 643-644, 1979.

9) April RS, Vansonnenberg E. A case of neuromyelitis optica (Devic's syndrome) in systemic lupus erythematosus. Clinicopathologic report and review of the literature. Neurology 26: 1066-1070, 1976.

10) Mok CC, Lau CS, Chan EY, Wong RW. Acute transverse myelopathy in systemic lupus erythematosus: clinical presentation, treatment, and outcome. J Rheumatol 25: 467-473, 1998.

11) Andrianakos AA, Duffy J, Suzuki M, Sharp JT. Transverse myelopathy in systemic lupus erythematosus. Report of three cases and review of the literature. Ann Intern Med 83: 616-624, 1975.

12) Minagar A, Alexander JS, Fowler MR, Long AC, Kelley RE. Devic disease: clinical course, pathophysiology, and management. Pathophysiology 9: 33, 2002.

13) Lucchinetti CF, Mandler RN, McGavern D, et al. A role for humoral mechanisms in the pathogenesis of Devic's neuromyelitis optica. Brain 125: 1450-1461, 2002.

14) Wingerchuk DM, Hogancamp WF, O'Brien PC, Weinshenker BG. The clinical course of neuromyelitis optica (Devic's syndrome). Neurology 53: 1107-1114, 1999.

15) Hughes GR. Central nervous system lupus-diagnosis and treatment. J Rheumatol 7: 405-411, 1980.

16) Provenzale JM, Barboriak DP, Gaensler EH, Roberston RL, Mercer B. Lupus-related myelitis: serial MR findings. Am J Neuroradiol 15: 1911-1917, 1994

17) Yazawa S, Kawasaki S, Ohi T, et al. Development of severe longitudinal atrophy of thoracic spinal cord following lupus-related myelitis. Intern Med 40: 353-357, 2001. 